OPEN ACCESS

Edited by:

Marcelo Menossi Menossi, Universidade Estadual de Campinas,

Brazil

Reviewed by:

Taras P. Pasternak, Albert Ludwig University of Freiburg,

Germany

Antonio Ferrante,

Università degli Studi di Milano, Italy

*Correspondence: Yali Zhang

zhangyali@cau.edu.cn

Specialty section:

This article was submitted to

Plant Biotechnology,

a section of the journal Frontiers in Plant Science

Received: 05 March 2017 Accepted: 02 June 2017

Published: 20 June 2017

Citation:

Cai Y, Tu W, Zu Y, Yan J, Xu Z, Lu J and Zhang $Y$ (2017) Overexpression of

a Grapevine Sucrose Transporter

NvSUC27) in Tobacco Improves Plant

Growth Rate in the Presence of

Sucrose In vitro.

Front. Plant Sci. 8:1069.

doi: 10.3389/fpls.2017.01069

\section{Overexpression of a Grapevine Sucrose Transporter (VvSUC27) in Tobacco Improves Plant Growth Rate in the Presence of Sucrose In vitro}

\author{
Yumeng Cai ${ }^{1}$, Wenrui Tu ${ }^{1}$, Yunyun $\mathrm{Zu}^{1}$, Jing Yan ${ }^{1}$, Zimo $X u^{1}$, Jiang $L u^{1,2}$ and Yali Zhang ${ }^{1 *}$ \\ ${ }^{1}$ Beijing Advanced Innovation Center for Food Nutrition and Human Health, College of Food Science and Nutritional \\ Engineering, China Agricultural University, Beijing, China, ${ }^{2}$ Center for Viticulture and Enology, School of Agriculture and \\ Biology, Shanghai Jiao Tong University, Shanghai, China
}

The import of sugar from source leaves and it further accumulation in grape berries are considerably high during ripening, and this process is mediated via sucrose transporters. In this study, a grape sucrose transporter (SUT) gene, VVSUC27, located at the plasma membrane, was transferred to tobacco (Nicotiana tabacum). The transformants were more sensitive to sucrose and showed more rapid development, especially roots, when cultured on MS agar medium containing sucrose, considering that the shoot/root dry weight ratio was only half that of the control. Moreover, all transformed plants exhibited light-colored leaves throughout their development, which indicated chlorosis and an associated reduction in photosynthesis. The total sugar content in the roots and stems of transformants was higher than that in control plants. No significant difference was observed in the leaves between the transformants and control plants. The levels of growth-promoting hormones were increased, and those of stress-mediating hormones were reduced in transgenic tobacco plants. The qRT-PCR analysis revealed that the expression of VVSUC27 was 1,000 times higher than that of the autologous tobacco sucrose transporter, which suggested that the markedly increased growth rate of transformants was because of the heterogeneously expressed gene. The transgenic tobacco plants showed resistance to abiotic stresses. Strikingly, the overexpression of VVSUC27 leaded to the up regulation of most reactive oxygen species scavengers and abscisic acid-related genes that might enable transgenic plants to overcome abiotic stress. Taken together, these results revealed an important role of VvSUC27 in plant growth and response to abiotic stresses, especially in the presence of sucrose in vitro.

Keywords: grapevine, VvSUC27, growth, abiotic stresses, sucrose

\section{INTRODUCTION}

Plants produce carbohydrate metabolites via photosynthesis to support their development. Fixed carbon is subsequently distributed to many tissues, e.g., developing leaves, flowers, fruits, and roots, which cannot reduce carbon alone. In most plants, sucrose is the predominant form of carbon transported through the vascular system to the sink organs. Therefore, sucrose transporters have a pivotal role in the translocation of sucrose. 
Since the first sucrose transporter (SUT) was isolated from spinach (Riesmeier et al., 1992), many clones belonging to this gene family have been isolated from various species and plant organs (Derrer et al., 2013). Some of these clones have been shown to be functional SUTs by expressing them in Xenopus oocytes or yeast (Deol et al., 2013; Oner-Sieben et al., 2015; Zanon et al., 2015), but their ability to regulate plant metabolism remains unclear.

One method used to elucidate the function of sugar transporters and their physiological roles in plants is to generate plants that have modified transporters (by sense overexpression, antisense inhibition, or gene disruption). Transgenic plants expressing antisense SUTs in potato (Kuhn et al., 1996; Lemoine et al., 1996; Gabriel-Neumann et al., 2011), tobacco (Burkle et al., 1998), or tomato (Bitterlich et al., 2014) have been characterized for their accumulation of leaf carbohydrates and impaired root development. Moreover, reduced tuber yield in potato lines, delayed or impaired flowering in tobacco lines, and increased mycorrhizal colonization in tomato lines have been observed. These results suggest that the antisense expression of SUTs in plants severely reduces plant growth, and that SUTs in the phloem are essential for carbohydrate partitioning, at least in tobacco, potato, and tomato (members of Solanaceae). Further, studies have investigated the effects of sucrose transporter overexpression. The overexpression of the Hordeum vulgare SUT (HvSUT1) increased grain protein content and deregulated the metabolic status of wheat (Triticum aestivum) grains (Weichert et al., 2010). Potato plants transformed with a sense spinach SUT (SoSUT1) showed reduced sucrose levels in leaves and varied tuber morphology (Leggewie et al., 2003). Transgenic potato plants with constitutive overexpression of SUT1 exhibited increased mycorrhization (Gabriel-Neumann et al., 2011). The overexpression of a potato SUT (StSUT1) in pea cotyledon storage parenchyma cells enhanced the sucrose uptake capacity of cotyledons and their growth rates (Rosche et al., 2002). However, this enhancement was achieved in a single organ, rather than in the entire plant.

Three putative SUT (VvSUC11, VvSUC12, and VvSUC27) cDNAs have been identified in grape (Vitis) berry (Davies et al., 1999). Their abilities to transport sucrose have been shown in a heterologous yeast expression system; considering the value of $K_{m}$ to sucrose, VvSUC11 and VvSUC12 are intermediate-affinity SUTs ( $K_{m}$ of 0.88 and $1.36 \mathrm{mM}$, respectively; Ageorges et al., 2000; Manning et al., 2001), and VvSUC27 is a low-affinity/highcapacity (LAHC) sucrose transporter $\left(K_{m}\right.$ of $8.0-10.5 \mathrm{mM}$; Zhang et al., 2008). Northern blot analysis was used to determine the expression of these three SUTs in different tissues; $V v S U C 11$ and $V v S U C 12$ were found to be expressed in numerous tissues, except in roots and tendrils. However, VvSUC27 was highly expressed in the roots, petioles, stems, young leaves, and tendrils and was expressed at lower levels in berries and old leaves. Thus, VvSUC27 is a sink-specific gene, which is responsible for phloem loading and sugar retrieval during long-distance transport and is closely related to members of the SUT1 subfamily (Davies et al., 1999; Afoufa-Bastien et al., 2010). However, during berry development, the transcript abundance of VvSUC27 was lower than that of the other two SUTs assessed (VvSUC11 and VvSUC12); the decreased transcript abundance was exhibited especially after veraison (Davies et al., 1999; Deluc et al., 2007; Afoufa-Bastien et al., 2010; Pastenes et al., 2014; Xu et al., 2015). Notably, a small portion of plasmodesmata was apparently blocked in the ripening stage, and the phloem strands were blocked during the late stage (Zhang et al., 2006). These phenomena might be associated with a shift in phloem unloading from the symplasmic to apoplasmic pathway during berry development. However, studies focusing on the function of VvSUC27 are not systematic, and the effect of increased VvSUC27 activity on plant properties has not been determined. Previously, we isolated and characterized VvSUC27 in a heterologous yeast strain to characterize its function as a LAHC SUT; its sucrose uptake activity was activated in transformed yeast by monosaccharides and inhibited by maltose and diethyl pyrocarbonate (Zhang et al., 2008). In this study, in order to further investigate the function and effect of VvSUC27 on a genetically modified plant phenotype and the related mechanisms, we determined the gene expression pattern and subcellular localization of VvSUC27 and generated transformants. Our results indicated that $V v S U C 27$ is a candidate gene to improve plant growth and abiotic stress tolerance in vitro, especially in the presence of sucrose.

\section{MATERIALS AND METHODS}

\section{Plant Material and Culture Condition}

Grapevine berry of $V$. riparia DVIT1848, Frontenac ( $V$. riparia $\times$ Landot 4511), V. amurensis Ruper. "Zuoshan-1," V. amurensis Ruper. "Zuoshan-2," and Vitis vinifera L. "Cabernet Sauvignon" were collected from Shangzhuang (Beijing, China). V. vinifera L. "Cabernet Sauvignon" was collected about 20 weeks postflowering.

The culture medium was solid Murashige and Skoog (MS) medium (not containing sucrose). The plant material used was grown on agar MS with or without $30 \mathrm{~g} \cdot \mathrm{L}^{-1}$ sucrose. All plants were grown in vitro at a constant temperature of $25^{\circ} \mathrm{C}$ with a $16 / 8$-h day/night regime $\left(150 \mu \mathrm{moL} \cdot \mathrm{m}^{-2} \cdot \mathrm{s}^{-1}\right)$.

\section{Total RNA Isolation}

Total RNA was isolated as previously described with some modifications (Zhang et al., 2008). Fresh tissue was cut from live plants, frozen in liquid nitrogen, and stored at $-80^{\circ} \mathrm{C}$. RNA was isolated by grinding $0.2 \mathrm{~g}$ of tissue to a fine powder with a mortar and pestle pre-chilled with liquid nitrogen. The powder was transferred to a warm cuvette containing $0.7 \mathrm{~mL}$ of extraction buffer (2\% CTAB, 2\% PVP, 25 mM EDTA, 100 mM Tris-HCl, $\mathrm{pH}$ 8.0, $2.0 \mathrm{M} \mathrm{NaCl}$ ) and $14 \mu \mathrm{L}$ of $\beta$-mercaptoethanol and ground for $30-45 \mathrm{~s}$ to thoroughly mix the material. The homogenate was incubated in a $65^{\circ} \mathrm{C}$ water bath for $10 \mathrm{~min}$, and then extracted with an equal volume of chloroform/isoamyl alcohol (24:1), shaked. The two layers were separated by centrifugation at 13,000 $\times \mathrm{g}$ for $15 \mathrm{~min}$. The aqueous phase was collected and extracted with an equal volume of chloroform/isoamyl alcohol (24:1) again, centrifuged at $13,000 \times \mathrm{g}$ for $15 \mathrm{~min}$. The aqueous phase was collected and the total RNA was precipitated with a $1 / 4$ volume of $10 \mathrm{M} \mathrm{LiCl}$ at $-20^{\circ} \mathrm{C}$ for $6 \mathrm{~h}$. The nucleic acids were collected by centrifugation at $13,000 \times \mathrm{g}$ for $30 \mathrm{~min}$ at $4^{\circ} \mathrm{C}$ and then 
rinsed with $70 \%$ ethanol once. After the pellet was dried, it was resuspended in $100 \mu \mathrm{L}$ DEPC water.

\section{Isolation of VvSUC27 cDNA}

Fresh grape tissue was cut from live plants, frozen in liquid nitrogen, and stored at $-80^{\circ} \mathrm{C}$ for total RNA preparation. Total RNA was isolated from $V$. vinifera L. "Cabernet Sauvignon" as described. The FastQuant RT Kit (with gDNase; Tiangen, China) was used for the synthesis of cDNA as per manufacturer's instructions. Oligo dT was used as the primer for cDNA synthesis. The cDNA was designed with respect to the cDNA encoding VvSUC27 (AF021810; Davies et al., 1999). The oligonucleotide probes used were as follows:

\section{5'-ATGGAGTTAGCCAAGCCTTCTTC-3' \\ 5' -TTAAGACGACGGCTGAGTCCTC-3'}

These probes were used to generate a clone with a full-length 1,518-bp PCR product, which contained the sequence of the $V v S U C 27$ gene. The fragment was purified and ligated into a pGEX-T vector (Promega).

\section{Preparation of Transgenic Plants}

The binary vector $\mathrm{pBI} 121$ was used to carry two cassettes between the T-DNA borders (Nowak et al., 2001): (i) the npt-II gene conferring kanamycin resistance to the transformants; and (ii) the glucuronidase (GUS) gene, whose transcription is under the control of the $35 \mathrm{~S}$ promoter. In our study, any side-effect due to the GUS gene were avoided by replacing it by the VvSUC27 gene. The $V v S U C 27$ gene was inserted in sense orientation by using the BamHI and SacI. Plasmid integrity was verified using restriction enzyme analysis. The binary vectors were transferred to Agrobacterium tumefaciens LBA 4404, and plants were transformed using the leaf disc transformation procedure (Graybosch et al., 1989). Tobacco (N. tabacum "Samsun") plants were grown on sterilized MS medium at $25^{\circ} \mathrm{C}$, with a 16 -h photoperiod. Rooted plants from sterile cultures were transferred to pots containing sterilized garden soil and grown under an 11$\mathrm{h}$ photoperiod at $25^{\circ} \mathrm{C}$. The plants were allowed to flower and self-fertilize, and the seeds were collected. Homozygotes of $\mathrm{T}_{3}$ seedlings were used for further analyses.

\section{DNA Isolation and Southern Blotting}

Genomic DNA was isolated from the supernatant of LiCl RNA precipitation by adding 2 volumes of ethanol, followed by centrifugation at $5,000 \times \mathrm{g}$ for $15 \mathrm{~min}$. The DNA pellet was resuspended in water and treated with RNase $\left(1 \mathrm{~g} \cdot \mathrm{L}^{-1}\right)$ and proteinase $\mathrm{K} 20\left(50 \mathrm{mg} \cdot \mathrm{L}^{-1}\right)$ for $60 \mathrm{~min}$ at $37^{\circ} \mathrm{C}$. Following extraction with phenol-chloroform-isoamyl alcohol (25:24:1, by volume), the DNA was precipitated by the addition of isopropanol. The pellet was washed with $70 \%$ ethanol and dissolved in water. The DNA (7-10 $\mu \mathrm{g})$ was digested with BamHI and SacI for $16 \mathrm{~h}$. The DNA fragments were separated on a $0.8 \%$ agarose gel overnight at $30 \mathrm{~V}$ and transferred onto Hybond $\mathrm{N}^{+}$membranes (Amersham Life Science). Southern blot was performed using the DIG High Prime Labeling and Detection Starter Kit (Roche) as described by the manufacturer.

\section{Uptake Activity}

Uptake experiments were performed using 8-week-old tobacco (N. tabacum "Samsun") plants. The previously reported method was used for reference (Leterrier et al., 2003); however, because a different transporter was investigated in this study, sucrose was used as the substrate. The incubation medium contained $1 \mathrm{mM}$ sucrose and $0.1 \mathrm{MBq} \mathrm{mL}^{-1}$ sucrose- $\left[{ }^{14} \mathrm{C}\right]$ (Amersham Pharmacia Biotech, Buckinghamshire, UK). The sucrose concentration was determined using the $K_{m}$, which is usually determined for plant SUTs in the plasma membrane vesicles (0.2-1 mM; Lemoine, 2000), and is expected to saturate the transporter without any strong contribution by a diffusional component.

\section{Localization Study}

The VvSUC27 for subcellular localization was cloned by PCR amplifying the open reading frame (ORF) of its cDNA. The primers used are shown in Table S1. The pUC19-CaMV 35SVvSUC27-GFP plasmid was used for transient expression in Arabidopsis protoplasts. Protoplasts were isolated from the leaves of 3- to 4-week-old plants of Arabidopsis (ecotype Columbia), transiently transformed using polyethylene glycol, and incubated at $23^{\circ} \mathrm{C}$ for $16 \mathrm{~h}$ (Ueda et al., 2001). We used the fusion plasmid pBI121-CaMV 35S-VvSUC27-GFP, which was expressed transiently in Nicotiana benthamiana via agroinfiltration to determine the subcellular localization of VvSUC27 as previously described (Xiang et al., 2016). Green fluorescent protein (GFP) fluorescence was observed using a Nikon C1 Si/TE2000E confocal laser scanning microscope, and the EZ-C1 3.00 software was used for image processing.

\section{Seedling Growth Experiments}

Seedling growth experiments were performed using solid MS media containing sucrose concentrations ranging from 0 to $6 \%$ $\left(\mathrm{g} \cdot 100 \mathrm{~mL}^{-1}\right)$. The seedling growth ratio $(\%$, the number of germinated seeds/the number of whole seeds in each bottle) of the transformed tobacco seeds on media containing different sucrose concentrations was investigated for $9 \mathrm{~d}$. The inhibition rate of each line was calculated as follows:

$$
I R=\frac{\mathrm{SRx}-\mathrm{SRo}}{\text { SRo }} \times 100 \%,
$$

Where $\mathrm{SR}_{x}$ and $\mathrm{SR}_{o}$ represent the seedling growth ratio on solid MS media containing the corresponding sucrose concentration and that without sucrose, respectively.

\section{Growth Conditions and Phenotype Analysis}

Plants were grown in a greenhouse at a constant temperature of $25^{\circ} \mathrm{C}$ with a $16 / 8$-h day/night regime $\left(150 \mu \mathrm{moL} \cdot \mathrm{m}^{-2} \cdot \mathrm{s}^{-1}\right)$ on MS medium containing $0.7 \%(\mathrm{w} / \mathrm{v})$ agar ( $\mathrm{pH} 5.8)$ in the absence and presence of $30 \mathrm{~g} \cdot \mathrm{L}^{-1}$ exogenous sucrose. Seeds from each transformant and control (CK) were planted on MS medium supplemented with or without $30 \mathrm{~g} \cdot \mathrm{L}^{-1}$ exogenous sucrose in 200 $\mathrm{mL}$ culture vessels with $12 \mathrm{~cm}$ height $(50 \mathrm{~mL}$ medium in each vessel). Thirty-day-old seedlings were used to determine the stem diameter, leaf number, leaf area, and root number. Stem length was recorded every week for 7 weeks. For stress resistance assay, 





seeds were planted on the medium supplemented with $0.15 \mathrm{M}$ $\mathrm{NaCl}$ or $0.2 \mathrm{M}$ mannitol.

\section{Dry Weight Determination}

Four-to-six transformed tobacco plants for each independent clone were cultured in vitro for 6 weeks. Roots, leaves, and stems of each plant were cut and weighed separately after 24-h incubation at $80^{\circ} \mathrm{C}$.

\section{Sugar Measurements}

Fresh leaves, stems, and roots were separated from the plants, placed at $105^{\circ} \mathrm{C}$ for $15 \mathrm{~min}$, and then dried at $75^{\circ} \mathrm{C}$ overnight until a constant weight was reached. The dry tissue was ground using a mortar and pestle. The dry powder $(25 \mathrm{mg})$ was dissolved in $1.5 \mathrm{~mL} 80 \%$ ethanol and distilled at $80^{\circ} \mathrm{C}$ for $30 \mathrm{~min}$, modified anthrone-sulfuric acid method for further determination. Measurements were performed as previously described (Somani et al., 1987; Zhang et al., 2016).

\section{Determination of Endogenous Phytohormone Content}

Seven-week-old seedlings were collected following planting on MS medium with or without $30 \mathrm{~g} \cdot \mathrm{L}^{-1}$ exogenous sucrose supplementation. Endogenous methyl jasmonic acid (MeJA), salicylic acid (SA), indole-3-acetic acid (IAA), abscisic acid (ABA), and gibberellic acid $\left(\mathrm{GA}_{3}\right)$ were extracted from the transformed (Lines 9, 15, and 16) and CK as described previously (Pan et al., 2010) and measured using a high-performance liquid chromatography-mass chromatography system (HPLCMS) (Varian, USA) by the Beijing Center for Physical and Chemical Analysis.

\section{Stress Growth Conditions and Phenotype Analysis}

For the stress resistance assay, seeds were planted on $0.7 \%$ (w/v) agar MS medium ( $\mathrm{pH}$ 5.8) supplemented with $0.15 \mathrm{M}$ $\mathrm{NaCl}$ or $0.2 \mathrm{M}$ mannitol and no exogenous sucrose or $30 \mathrm{~g} \cdot \mathrm{L}^{-1}$ exogenous sucrose under a 16/8-h day/night regime $(150 \mu \mathrm{moL}$ $\left.\mathrm{m}^{-2} \mathrm{~s}^{-1}\right)$. For the light experiment, seedling growth in MS media containing $30 \mathrm{~g} \cdot \mathrm{L}^{-1}$ sucrose was investigated for $30 \mathrm{~d}$, after which they were placed under low light $\left(75 \mu \mathrm{moL} \mathrm{m}{ }^{-2} \mathrm{~s}^{-1}\right)$ or no light conditions for further $30 \mathrm{~d}$. All plants were grown in a greenhouse at a constant temperature of $25^{\circ} \mathrm{C}$. Stem diameter, stem length, leaf number, leaf area, and root number were measured.

\section{Quantitative Real-Time PCR}

RNA was extracted from four kinds of frozen grapes or plant leaves, stems, and roots of $\mathrm{CK}$ and the three overexpression lines; the samples were treated with DNase as described above. Quantitative real-time PCR (qRT-PCR) was performed using SYBR Premix Ex Taq (TAINGEN, China) by using appropriate gene-specific primers (Table S1). The threshold cycle for each PCR with different concentrations of cDNA was determined and compared against a standard DNA $(E F 1 \alpha)$ that was also analyzed simultaneously (Wang et al., 2014). A ratio of the concentration of gene-specific mRNAs in the sample was subsequently calculated.

\section{Statistical Analyses}

One-way analysis of variance (ANOVA) and a subsequent Tukey's test were performed to analyze data by using SPSS16.0 (SPSS Corp., Chicago, IL, USA). A $P<0.05$ was considered statistically significant. Relationships between $V v S U C 27$ expression and sugar accumulation were explored using Pearson's correlation co-efficient. Pearson's $r$ was always between -1 and +1 , where -1 refers to a perfect negative relationship; +1 , to a perfect positive relationship; and 0 , to the absence of a relationship. All experiments were repeated three times.

\section{RESULTS}

\section{VvSUC27 Expression is Negatively Correlated with Sugar Accumulation}

The relationship between VvSUC27 gene expression and sugar accumulation was investigated by isolating total RNA and sugars from grape berry tissue during berry ripening. Sugar content was relatively low in the beginning and markedly increased after veraison (Figures 1A-D). In contrast, a relatively high level of VvSUC27 expression was noted in berries before veraison. However, after veraison, its level remarkably decreased. The data obtained from four different varieties of grape suggested a negative correlation between $V v S U C 27$ gene expression and sugar accumulation. This finding was confirmed by conducting a correlation analysis (Figure 1E). A strong negative correlation was noted between VvSUC27 gene expression and sugar accumulation, suggesting that VvSUC27 was probably responsible for sugar retrieval during longdistance transport. Therefore, we selected this gene for further study.

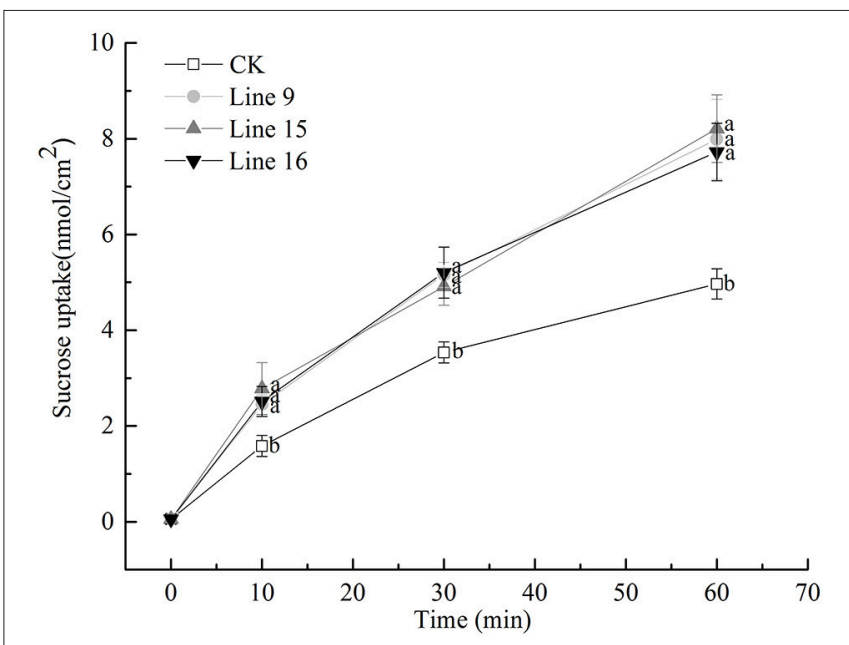

FIGURE 2 | Uptake rate of sucrose- $\left[{ }^{14} \mathrm{C}\right](1 \mathrm{mM})$ by leaf discs of VvSUC27-transformed tobacco plants. Data are expressed as the mean \pm $S D$ of determinations from three independent samples. Analysis for each sample was repeated three times, and similar results were obtained. Different letters indicate significant differences $(P<0.05)$ differences between transformants (Lines 9, 15, and 16) and CK, as determined by one-way analysis of variance followed by Tukey's test using SPSS statistical software. 


\section{Cloning of VvSUC27 into an Expression Vector and Transgenic Tobacco Production}

Total RNA was isolated from grape berry tissue, VvSUC27 (AF021810) cDNA was amplified using RT-PCR, and a 1518bp fragment was cloned into the pGEM-T easy vector. After the vector was double-digested with $\mathrm{BamHI} / \mathrm{SacI}$, the coding region was ligated to the binary vector pBI121 in the sense orientation (Figure S1A).

The construct and blank plasmid were used to transform tobacco leaf discs by using the Agrobacterium-mediated transformation method. Fifteen lines that showed positive results in Southern blot analysis (Figure S1B) were cultured on antibiotic-selection medium until new roots and shoots emerged; they were transferred to the greenhouse for growth until seeds were produced. The primary tobacco transformants $\left(\mathrm{T}_{0}\right)$ grown from the transformed leaf discs expressing $V v S U C 27$ cDNA were selfed to generate the $T_{1}$ generation. Phenotypic differences between the transformants and CK plants were not visible in the field. The homozygotes of $T_{3}$ seedlings were chosen for further analysis.

\section{VvSUC27 as a Functional SUT Is Located at the Plasma Membrane}

The function of VvSUC27 as a SUT was confirmed again by monitoring the uptake of sucrose- $\left[{ }^{14} \mathrm{C}\right]$ by the leaf tissues; all the transformants showed a significantly high capacity for sucrose uptake into source leaves (Figure 2), which indicated that VvSUC27 was located at the plasma membrane. The location of VvSUC27 in the plasma membrane was further confirmed by subcellular localization. The C-terminal end of VvSUC27 was fused to GFP and expressed under the control of the CaMV $35 \mathrm{~S}$ promoter [pUC19-CaMV 35S-VvSUC27-GFP or pBI121-CaMV 35S- VvSUC27-GFP] to determine its subcellular localization by monitoring GFP fluorescence in Arabidopsis protoplasts and $N$. benthamiana (Figure 3). In Arabidopsis protoplasts and $N$. benthamiana transformed with control vector [pUC19-GFP and

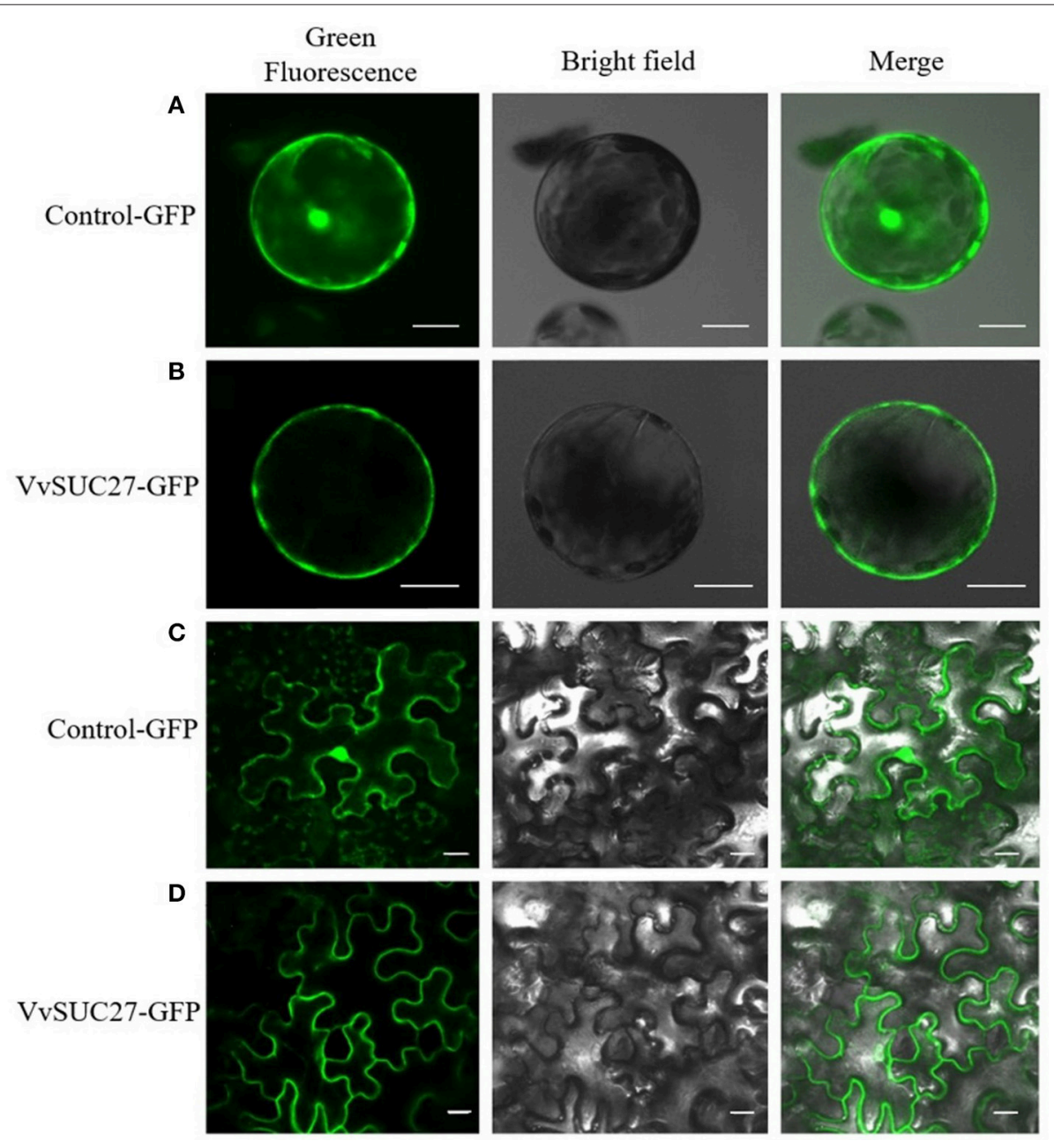

FIGURE 3 | Subcellular localization of VvSUC27 by transient expression of GFP fusion proteins in Arabidopsis protoplasts and Nicotiana benthamiana. Transient expression of GFP (as control) and VvSUC27-GFP under the control of the 35S promoter Arabidopsis protoplasts (A,B) and N. benthamiana (C,D). Localization of GFP to the plasma membrane, cytoplasm, and nucleus in Arabidopsis protoplasts (A) and N. benthamiana (C). Plasma membrane localization of VvSUC27 in Arabidopsis protoplasts (B) and N. benthamiana (D). Bars $=10 \mu \mathrm{m}$. 


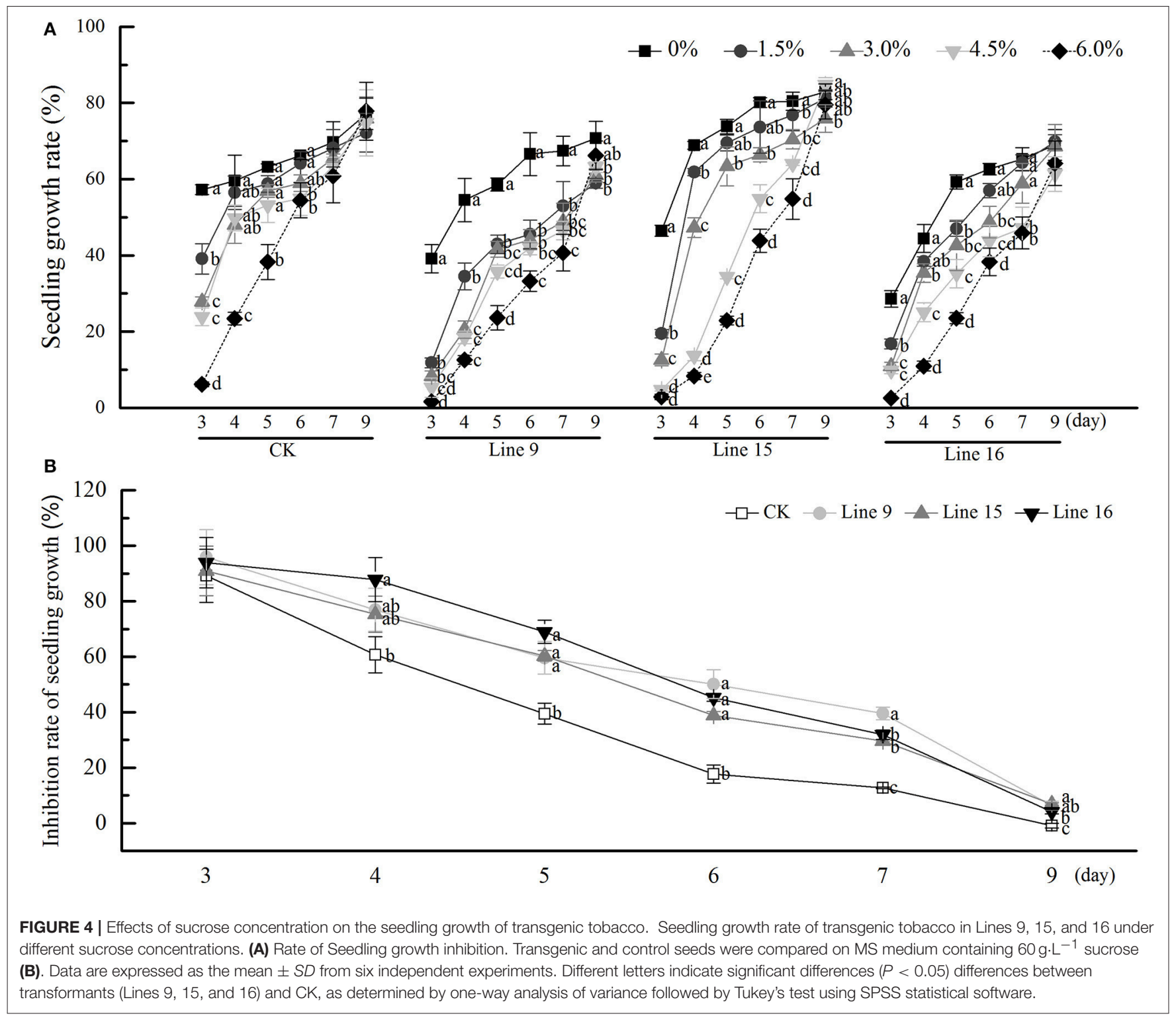

pBI121-GFP, respectively], green fluorescence was distributed in the cytoplasm, nucleus, and plasma membrane of the transgenic cells (Figures 3A,C). However, green fluorescence was exclusively detected in the plasma membrane of cells transformed with the fusion plasmid (Figures 3B,D). These results indicated that VvSUC27 was localized to the plasma membrane.

\section{VvSUC27 Overexpression Lines Show a Stronger Developmental Phenotype When Grown on Sucrose Medium}

We investigated the inhibitory effects of exogenous sucrose on the seedling growth of transgenic tobacco and found that higher sucrose concentrations resulted in a lower seedling growth rate, till day 9, relieved inhibition (Figure 4A). The inhibition rate of each line was compared with that of $\mathrm{CK}$ at a sucrose concentration of $6 \%$ (Figure 4B), and the transformants showed greater inhibition than CK. Then the seeds of transformants were sterilized and germinated on MS medium containing $30 \mathrm{~g} \cdot \mathrm{L}^{-1}$ sucrose. Compared with CK, transformants had root systems that were noticeably more developed, had taller and thicker stem, and included more and larger leaves (Figures 5A,B). Among the 15 transformant lines, only two lines (Lines 5 and 8) showed no visible phenotypic distinctions compared to CK (data not shown). The visible phenotype of transformants was shown not only in the remarkable improvement in performance, but also in terms of chlorosis observed in mature leaves (Figures 5C,D). This phenotype was observed after the third week. Almost all leaves (except cotyledons) of transgenic tobacco were yellow, and this phenotype was observed in all 13 transgenic lines and throughout their development in vitro (data not shown). The yellow region was mainly noted at the tips of mature leaves, indicating a reduction in photosynthesis. However, transformants grown on MS medium without sucrose, as in 

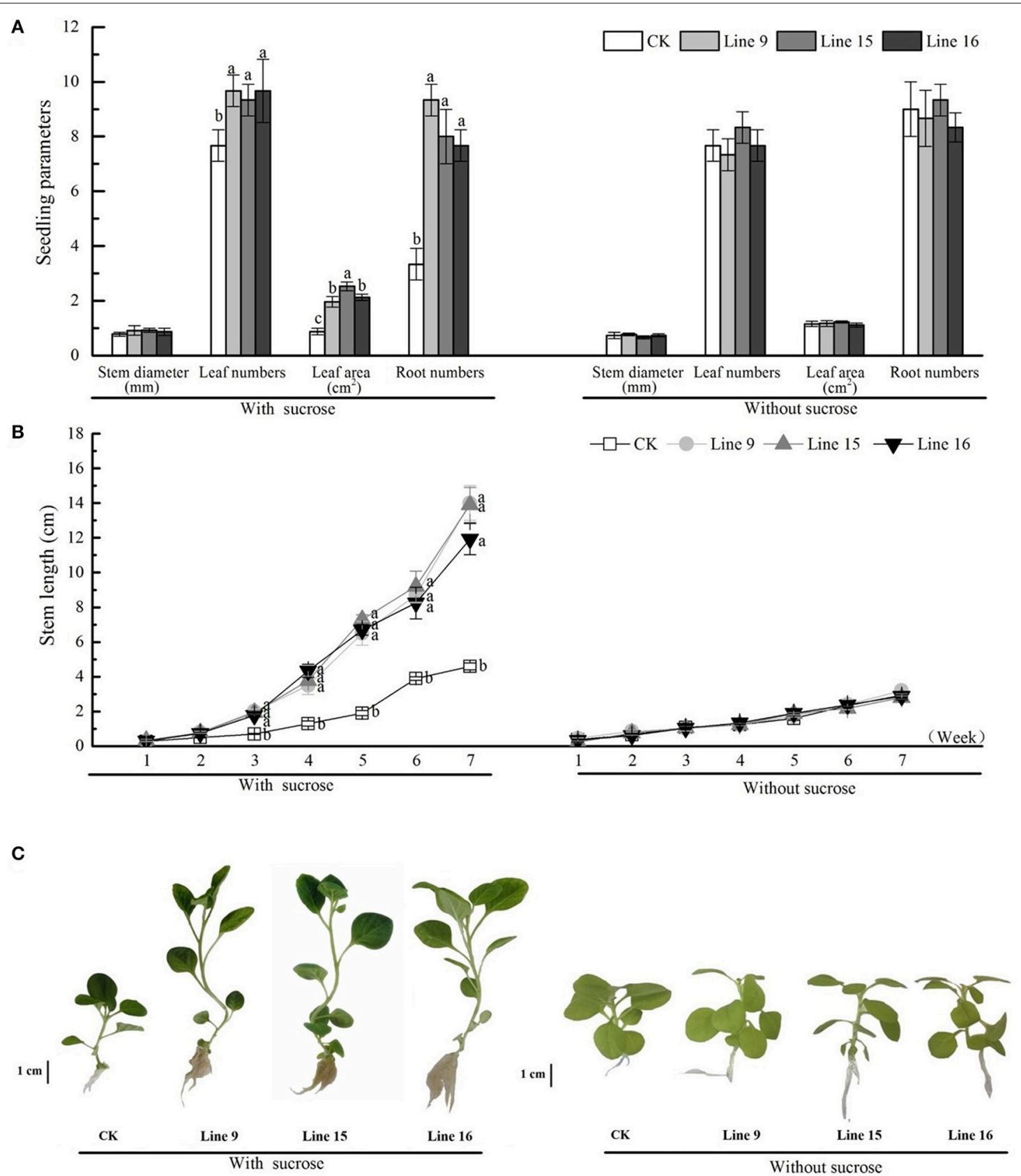

D

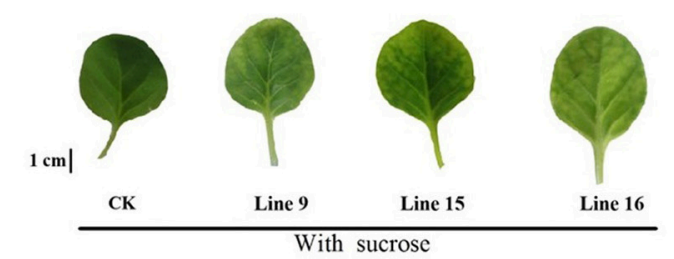

$1 \mathrm{~cm}$

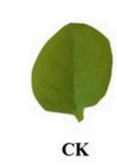

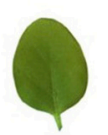

Line 9
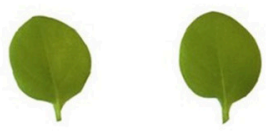

Line 16

Without sucrose

FIGURE 5 | Phenotypes of VvSUC27-transformed tobacco plants. The plants were grown in vitro on MS medium containing $30 \mathrm{~g} \cdot \mathrm{L}^{-1}$ sucrose or without sucrose. Thirty-day-old seedlings were used to investigate stem diameter, leaf number, leaf area, and root number (A). Time-course of stem growth in transgenic tobacco plants and CK grown in vitro. Stem length (between the root/shoot junction stem to the base of the growing apex) was measured over 7 weeks (B). Data are expressed as the mean $\pm S D$ from six independent experiments. Different letters indicate significant differences $(P<0.05)$ differences between transformants (Lines 9 , 15, and 16) and CK, as determined by one-way analysis of variance followed by Tukey's test using SPSS statistical software. Phenotypic development in 7-week-old seedlings of transformants compared with that in 7-week-old seedlings of CK (C). Leaves were cut from 7-week-old transformed plants and CK (D). 




FIGURE 6 | Dry weight and shoot/root ratio of VVSUC27-transformed tobacco plants. The plants were grown in vitro for 7 weeks after being plated on modified MS medium containing $30 \mathrm{~g} \cdot \mathrm{L}^{-1}$ sucrose or without sucrose. The dry weight of the total plant and different plant parts (leaves, stems, roots) (A), the dry weight/seedling ratio (B), and the shoot/root ratio (C) of transgenic tobacco plants (Lines 9, 15, and 16) and CK were measured. Data are expressed as the mean $\pm S D$ from six independent experiments. Different letters indicate significant differences $(P<0.05)$ differences between transformants $(L i n e s ~ 9,15$, and 16$)$ and CK, as determined by one-way analysis of variance followed by Tukey's test using SPSS statistical software.

the control condition, were visually indistinct from the control (Figure 5). A little difference in morphology was noted between their roots, stems, and leaves (Figures $\mathbf{5 A}, \mathbf{B}$ ), and the leaves were of the same color (Figures 5C,D).

The dry weight of the different parts of transformants grown on MS medium containing $30 \mathrm{~g} \cdot \mathrm{L}^{-1}$ sucrose was determined at 6 weeks after germination. At least six plants were obtained and separated into leaves, stems, and roots. The mean values for each line are shown in Figure 6A. Although, the significant differences existed between certain transgenic lines, they were all significantly different from CK, the weight of roots, stems, and leaves gained significantly, which resulted in the total plant dry weight markedly increased. The dry weight/seedling ratio of root and stem were significantly gained, while the ratio markedly decreased in leaves (Figure 6B). The shoot/root ratio indicated that transformants had a relatively large and strong root system (Figure 6C). Similar to that in CK, the dry weight of different transgenic plant parts and the shoot/root ratio of the plants grown on MS medium without sucrose were visually indistinct
(Figure 6). The mean dry weight values for leaves, stems, and roots in each plant (all three transformants and CK) were almost $4,0.7,0.55$, and $5 \mathrm{mg}$, respectively (Figure 6A). Differences in the dry weight/seedling and shoot/root ratio for each plant were very small (Figures 6B,C).

\section{VvSUC27 Overexpression Lines Have Altered Photosynthesis, Sugar Metabolism, and Endogenous Phytohormone Levels When Grown on Sucrose Medium}

The roots and leaves of VvSUC27 cDNA-transformed tobacco plants showed markedly different phenotypes. Their microstructure was analyzed by performing slice analysis on the root and leaf organs of both transformants and CK (Figure 7).

The roots of transformants had more cell layers, and each cell in the roots had larger volume in cross-section in the transformants than in CK (Figure 7A). Karyons (Figure 7A, indicated by red arrowheads) of the transformed cells were larger. 


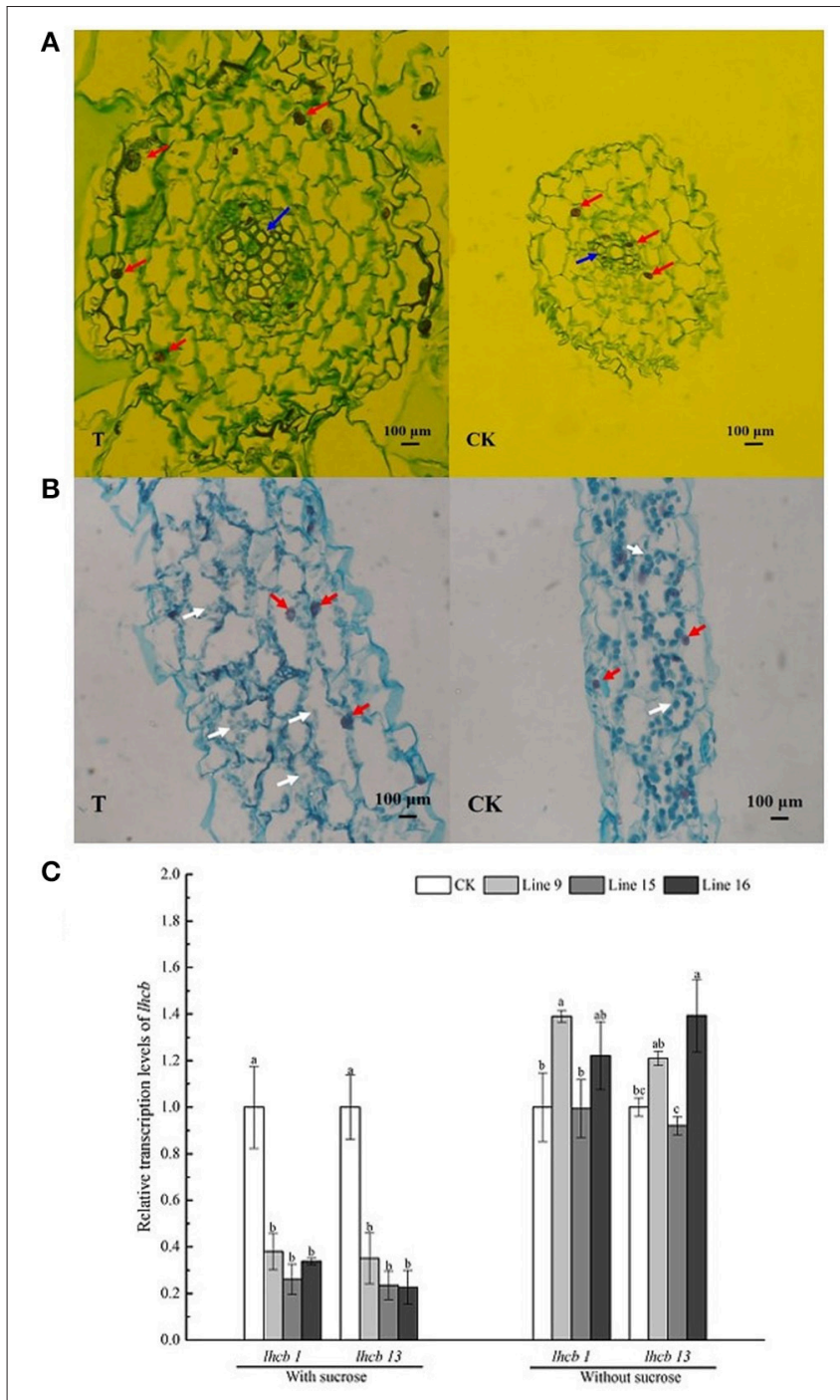

FIGURE 7 | Cross-section of the root and leaf and expression of chlorophyll-related genes in VvSUC27 transformants and CK. Organic slice analysis of root (A) and leaf (B). Organic slices were stained by Safranin $\mathrm{O} /$ Fast Green staining (SO/FG). Karyons, sieve element, and chloroplasts are indicated by red, blue, and white arrowheads, respectively. Transcript ratios of light harvesting chlorophyll a/b-binding protein $(I h c b)$ in transformants (Lines 9, 15, and 16) compared with that in CK (C). The plants were grown in vitro for 7 weeks after being plated on MS medium containing $30 \mathrm{~g} \cdot \mathrm{L}^{-1}$ sucrose. Data are expressed as the mean $\pm S D$ from six independent experiments. Different letters indicate significant differences $(P<0.05)$ differences between transformants (Lines 9, 15, and 16) and CK, as determined by one-way analysis of variance followed by Tukey's test using SPSS statistical software.

The most distinct section was the sieve element (Figure 7A, indicated by blue arrowheads), which was considerably more developed in the transformants. The leaves of transformants had numerous cells, but the chloroplasts in the leaves were of a smaller size (Figure 7B, indicated by white arrowheads). Furthermore, the expression of the photosynthetic gene light harvesting chlorophyll $a / b$ binding protein (lhcb) in the leaves was also investigated (Figure 7C; Kim et al., 2016). RNA isolated from CK was used as a negative control. Expression of the photosynthetic genes $l h c b 1$ and $l h c b 13$ were significantly inhibited in the leaves of all transformants. Further, the expression of the photosynthetic gene lhcb in transgenic leaves under control conditions (MS medium without sucrose) was unregulated; two lines exhibited higher expression among them only one line of each genes showed significant differences and one line exhibited slightly lower expression than that of CK.

Next, we intended to determine whether the overexpression of VvSUC27 also affected sugar metabolism. Different parts of the transgenic plants and CK (roots, stems, and leaves) were separated, and the carbohydrate contents of each part were measured (Figure 8). On the sucrose medium, the sugar content in the roots of transformants was significantly higher than that in CK. The total sugars in the roots of transformants were 2.7- (Line 9), 2.3- (Line 15), and 2.4-fold (Line 16) higher than those in the roots of CK. The sugar contents in the stems of transformants were also significantly higher than those in CK, however, except for the marked drop in sucrose content, no significant differences were observed in the content for other sugars in the leaves.

The major plant hormones include growth-promoting phytohormones GA, and IAA, as well as stress-mediating phytohormones ABA, JA, MeJA, and SA (Hossain et al., 2011; Trapp et al., 2014; Shahzad et al., 2015). In the present study, compared with CK, the VvSUC27 overexpressing lines planted on sucrose medium showed higher levels of growth-promoting phytohormones (all showed significantly higher $\mathrm{GA}_{3}$ levels; two lines showed significantly higher IAA levels) and lower levels of stress-mediating phytohormones (all lines showed significantly higher levels of ABA and MeJA, and one line showed significantly lower levels of SA; Figure 9). Under the control condition, the plants grown on no-sucrose medium showed no significant differences in phytohormone concentration (Figure 9).

\section{Changes in the Expression of NTSUT1 in VvSUC27 Overexpression Lines}

In our study, $V v S U C 27$ cDNA-transformed tobacco plants showed a change in their growth pattern and performance as well as their ability to acquire exogenous sucrose, with an increased sugar content observed in the roots and stems of transformants grown on the sucrose medium. When grown on the MS medium without sucrose, these changes were minimal; therefore, we investigated the expression of $V v S U C 27$ in transformants grown on MS medium with and without sucrose (Figure 10A). The expression of $V v S U C 27$ in all parts of transformants was high. Because sucrose is a very important signaling molecule in assimilate partitioning (Chiou and Bush, 1998), and several studies have shown that exogenous sucrose leads to a shift in the expression of SUTs in plants (Weber et al., 1997; Aoki et al., 1999; Matsukura et al., 2000), we investigated the expression of tobacco SUT (NtSUT1) in the different plant parts (root, stem, and leaf) of transformants by using real-time PCR (Figure 10B).

Lines 9, 15, and 16 exhibited the same expression patterns for all genes in all organs. RNA isolated from CK was also investigated as a negative control. When grown on the medium containing sucrose, NtSUT1 transcript levels were significantly 


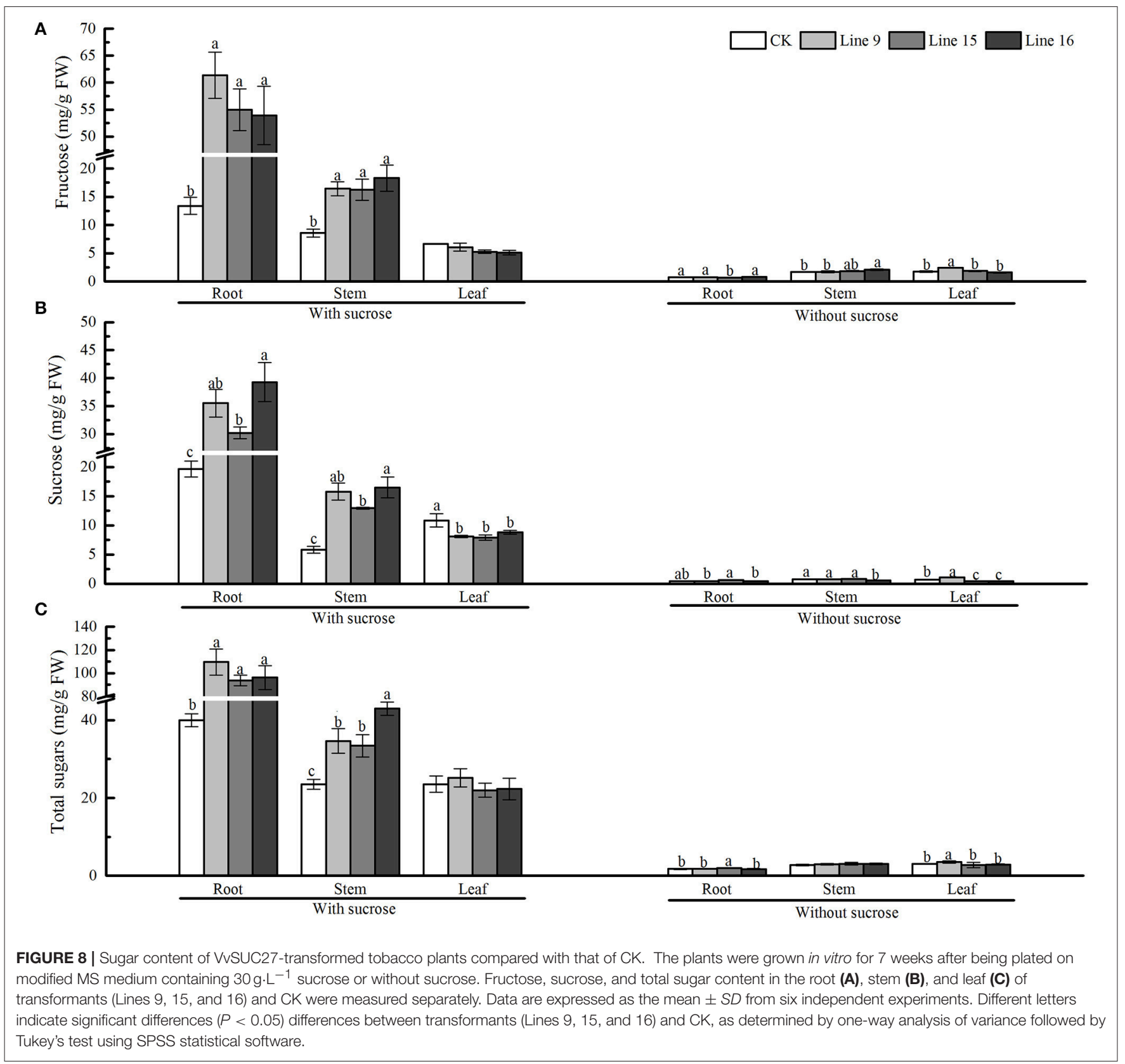

lower in the roots and leaves of transformants, whereas they were markedly increased in the stems. However, when grown on medium without sucrose, NtSUT1 transcript levels were increased in the roots and decreased in both the stems and leaves of transformants. Furthermore, NtSUT1 expression was significantly up-regulated in the same organ of the plants in the absence of exogenous sucrose.

\section{VvSUC27 Overexpression Lines Showed Increased Tolerance to Multiple Abiotic Stresses}

To explore the possible function of VvSUC27 in providing tolerance to abiotic stress in plants, we planted transformants and CK on medium with and without sucrose with (under salt and mannitol conditions) or without stress (phenotypes had been shown in Figure 5A). The transformants grown on both sucrose and no-sucrose MS medium showed a stronger phenotype to $\mathrm{CK}$ under stresses (Figure 11). We further investigated the transcriptional levels of nine reactive oxygen species (ROS) scavengers and ABA-related genes of transformants and $\mathrm{CK}$ grown under control or abiotic stress conditions (Figure 12 and Table S2). The genes in most of the transformants were significantly down-regulated under the control condition compared with that in CK (Figure 12A). Most of the genes in most VvSUC27 overexpression lines were upregulated after exogenous $\mathrm{NaCl}$ treatment (Figure 12B). While 


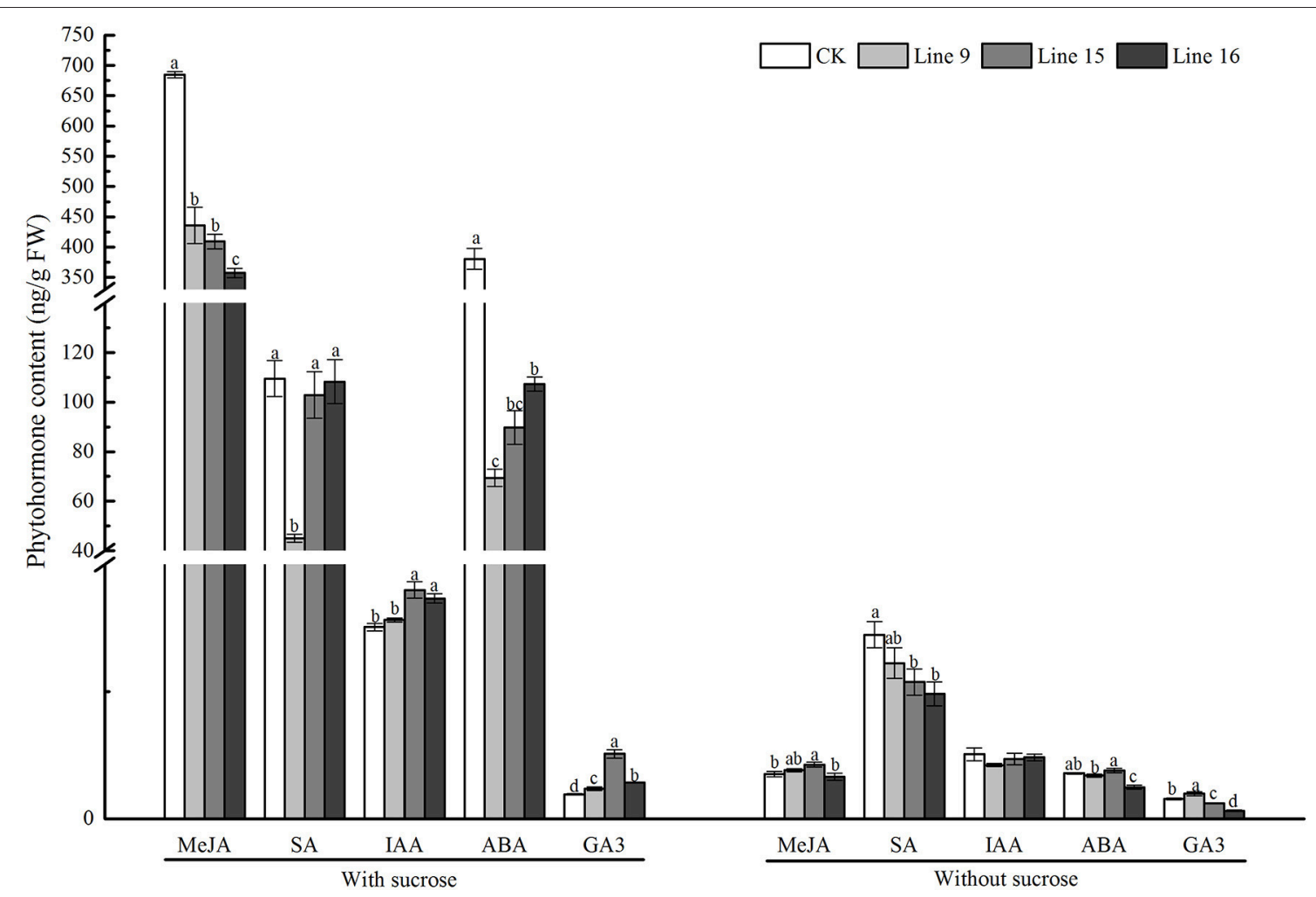

FIGURE 9 | Phytohormone content of VvSUC27-transformed tobacco plants compared with that of CK. The plants were grown in vitro for 7 weeks after being plated on modified MS medium containing $30 \mathrm{~g} \cdot \mathrm{L}^{-1}$ sucrose or without sucrose. Data are expressed as the mean $\pm S D$ from three independent experiments. Different letters indicate significant differences $(P<0.05)$ differences between transformants (Lines 9,15 , and 16$)$ and $C K$, as determined by one-way analysis of variance followed by Tukey's test using SPSS statistical software.

under exogenous mannitol treatment, almost all the genes in the transformants were significantly up-regulated (Figure 12C), especially on sucrose MS medium. Then, we compared all factors for each gene measured to find that most genes were down-regulated in $\mathrm{CK}$, while up-regulated in transgenic lines under stress than under the control condition. Most gene regulations presented significant differences, especially on sucrose MS medium (Table S2). The transformants also showed better performance compared to CK under low light and dark conditions when grown on the sucrose MS medium (Figure S2). The transformants had more roots, thicker and longer stems, and more and larger leaves. Moreover, the weaker the light, the longer and stronger were the stems and roots.

\section{DISCUSSION}

Sucrose is the primary product of photosynthetic $\mathrm{CO}_{2}$ fixation and is used for the distribution of assimilated carbon within higher plants. Transmembrane transport of sucrose requires the participation of SUTs. Although various studies have focused on SUTs of higher plants, relatively few have been performed on SUTs of $V$. vinifera, especially in terms of their function. In this study, VvSUC27, a SUT located at the plasma membrane, was found to show decreased transcript abundance during berry development and was involved in the promotion of seedling growth, sucrose absorption, sugar, and endogenous phytohormone distribution, and abiotic stress tolerance in the presence of MS medium containing exogenous sucrose.

\section{Transcript Abundance of VvSUC27 Decreases and Is Negatively Correlated with Sugar Accumulation during Berry Development}

In a previous study, VvSUC27 was described as a LAHC SUT, which might be responsible for phloem loading and sugar retrieval during long-distance transport, with a $K_{m}$ value for sucrose ranging between 8.0 and $10.5 \mathrm{mM}$ (Zhang et al., 2008; Afoufa-Bastien et al., 2010). Transcript analysis during berry development revealed that four varieties of $V$. vinifera showed relatively high expression levels of VvSUC27 in berries prior to veraison; however, after veraison, its expression in berries decreased remarkably (Davies et al., 1999; Deluc et al., 2007; Afoufa-Bastien et al., 2010; Pastenes et al., 2014; Xu et al., 2015). In this study, we used these four $V$. vinifera varieties to analyze the changes in the expression of $V v S U C 27$ and their relationship with sugar content 


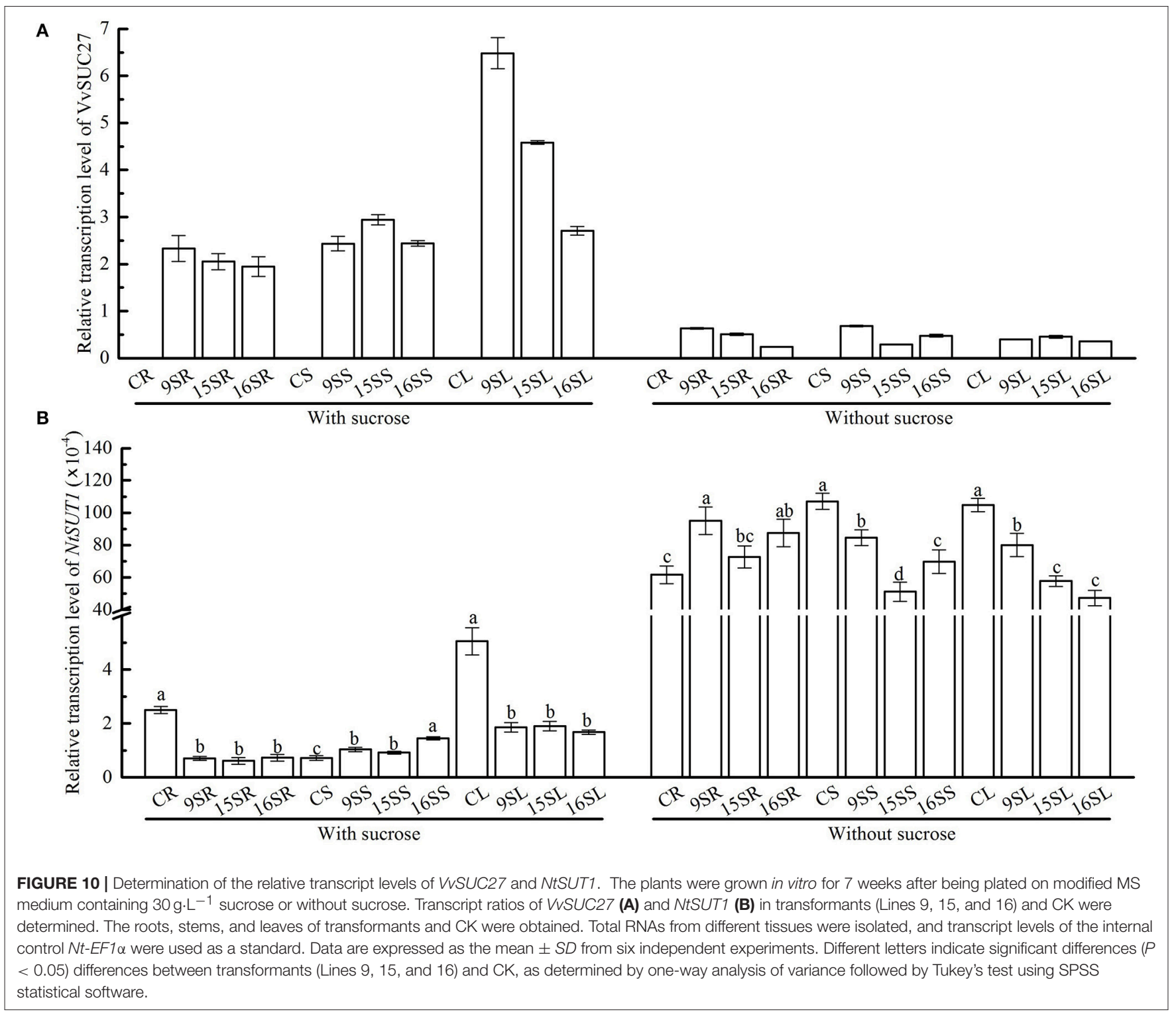

during berry development. With an increase in sugar content, the transcript abundance reduced remarkably during berry maturation (Figures 1A-D). Correlation analysis revealed a strong negative correlation (Figure 1B). The onset of ripening involves a shift in phloem unloading from the symplasmic to apoplasmic pathway, and, during that period, a small portion of plasmodesmata and phloem strands are apparently blocked in the ripening stage and during the late mature stage, respectively (Zhang et al., 2006). As an LAHC sinkspecific protein, VvSUC27 is responsible for phloem loading and sugar retrieval during long-distance transport (Zhang et al., 2008; Afoufa-Bastien et al., 2010). Therefore, we inferred that the decreasing transcript level of VvSUC27 might be consistent with the blocked plasmodesmata and phloem strands.
VvSUC27 Plays an Important Role in Improving the Absorption of Sucrose, Determining the Rate of Seedling Growth, and Promoting the Growth and Development of Transgenic Tobacco in the Presence of Exogenous Sucrose

As noted, VvSUC27 exhibited sucrose transport activity across the plasma membrane when expressed in yeast (Zhang et al., 2008). Sucrose-[ $\left.{ }^{14} \mathrm{C}\right]$ uptake (Figure 2) and subcellular localization (Figure 3) were successfully monitored in the present study. The total rate of sucrose uptake in transgenic plants containing VvSUC27 increased, indicating their enhanced ability for sucrose loading, which provides further evidence that VvSUC27 is a plasma membrane sucrose transporter. 




FIGURE 11 | VVSUC27 overexpression lines under abiotic stress. Phenotypic differences in terms of the leaf area, leaf number, elongation, and root number in MS media containing $30 \mathrm{~g} \cdot \mathrm{L}^{-1}$ sucrose or without sucrose under normal conditions, $\mathrm{NaCl}$, or mannitol from the start of the experiment for $30 \mathrm{~d}$. Different letters indicate significant differences $(P<0.05)$ differences between transformants (Lines 9, 15, and 16) and CK, as determined by one-way analysis of variance followed by Tukey's test using SPSS statistical software.

Sucrose can induce meristem quiescence as observed in the arrested development of seedlings germinated on high concentrations of sucrose (Lastdrager et al., 2014). Investigations on the effect of different sucrose concentrations on the growth of transgenic and CK seedlings showed that the higher the sucrose concentration, the stronger the rate of tobacco seedling growth inhibition, and the transformed plants were more sensitive to high sucrose concentrations (Figure 4). The inhibitory effects on early seedling development might be owing to osmotic stress, a hexokinase-independent mechanism, or ABA (Gibson, 2005). Interestingly, given sufficient time, the majority of seeds might germinate on even very high concentrations of sugars, which might be because of ABA metabolism (Laby et al., 2000; Cho and Yoo, 2011).

Many studies have suggested that reduced expression of sucrose transporters has deleterious effects on plant growth and development; however, improvement in plant performance in response to enhanced expression of the SUT has not yet been reported. Despite the higher protein content of HOSUT grains, no statistically significant effect was noted on wheat grains (Weichert et al., 2010). In our study, compared with plants in normal conditions, although the small volume of the vessel may inhibit the plant growth, resulting in smaller leaves, shorter stems, and weaker roots, there were still prominent phenotypic differences between transformants and CK (Figure 5), with VvSUC27-transformed plants exhibiting more rapid and stronger development. These enhanced plant morphologies resulted in a higher dry weight of the separate parts (leaves, stems, and roots) and a lower shoot/root ratio of the transgenic tobacco plants compared with that in CK (Figure 6). The decreased dry weight/seedling ratio of leaves and increased ratio of stems and roots in the transformants indicated that the main energy pathway switched from autotrophy to heterotrophy (Figure 6B). Transformants were also geminated under the same conditions used previously, except MS medium with no sucrose. Thus, the development of transformants was visually indistinct from that of CK (Figures 5, 6). We elucidated that sucrose uptake was the most important factor in the development of the enhanced phenotype.

\section{VvSUC27 Alters the Storage of Carbon and Phytohormones as well as the Expression of Endogenous Genes in Transgenic Tobacco in the Presence of Exogenous \\ Sucrose}

Sucrose is the main transported form of assimilates; however, it also regulates various processes such as carbon storage. 


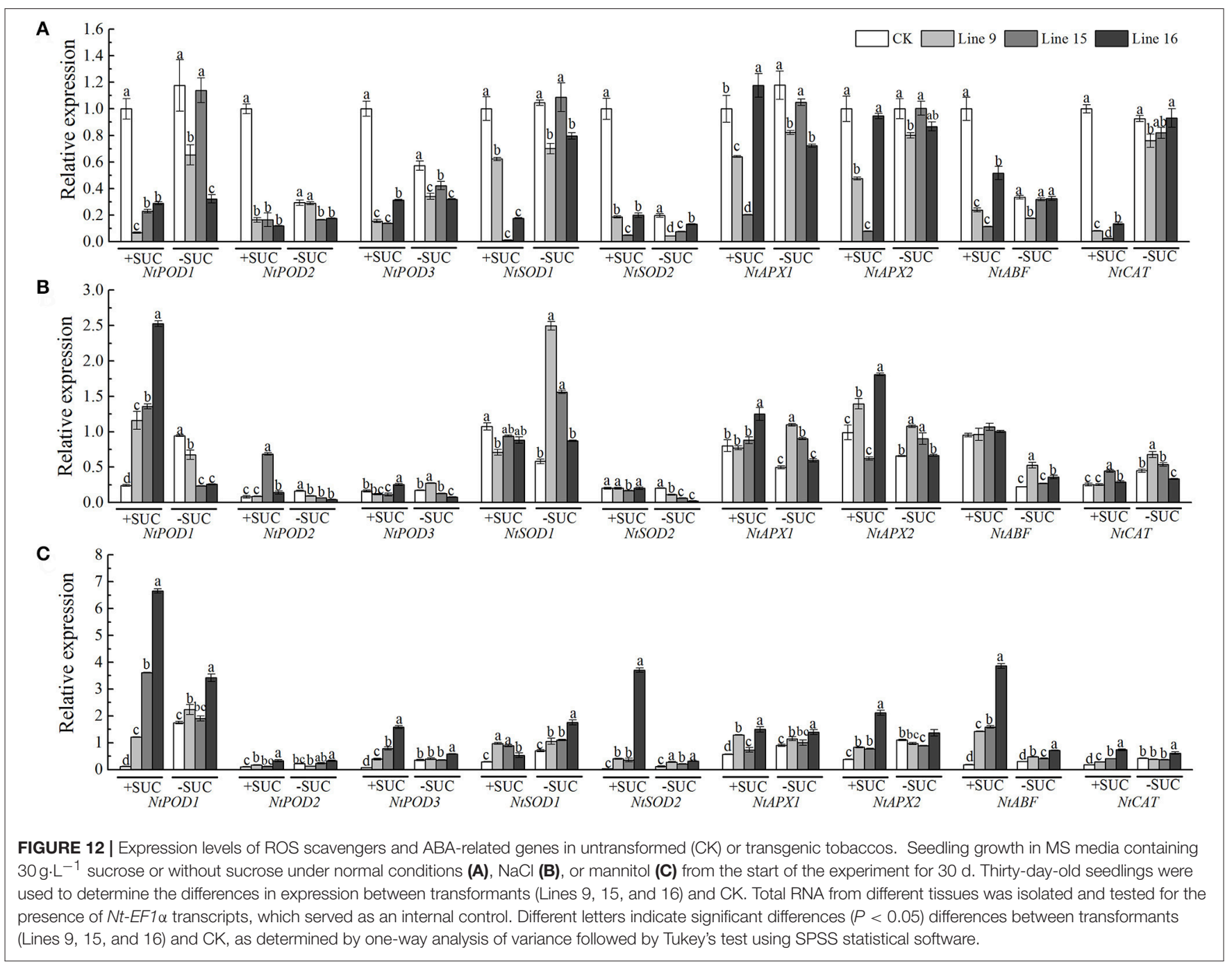

Carbohydrate accumulation in leaves leads to the downregulation of photosynthesis (Jang and Sheen, 1994). In our study, we planted transformants on sucrose-containing medium and found that the sieve elements were larger and more developed in the roots of transformants than in CK (Figure 7A), indicating the strong absorption and nutrient transport ability of the plants. The sucrose concentration did not increase in the leaves of transformants (Figure 8). However, the leaves changed their color to yellow, and chloroplasts in leaf cells were smaller and decreased in number compared with those in CK (Figure 7B), indicating that inactivate metabolism in the chloroplasts was the most likely reason for the observed chlorosis of the transformed leaves. Degeneration of chloroplasts indicated that the leaves of the transformants had impaired carbon fixation, resulting in reduced levels of carbohydrate metabolites fixed from the atmosphere. Furthermore, the expression of the photosynthetic gene $l h c b$ was significantly inhibited in the leaves (Figure 7C). The most important factor affecting photosynthetic capacity was the nitrogen absorption ability of roots, rather than the total non-structural carbohydrates (Sugiura et al., 2015), indicating that the high sucrose concentration might account for the high $\mathrm{C} / \mathrm{N}$ ratio of the whole plant and influence the nitrogen supply in roots, resulting in the inhibition of photosynthetic gene expression and changes in the photosynthetic capacity of the leaves. Our results showed that, when the roots of a transformed plant uptake sufficient carbohydrate, the plants restrain the function of the leaves to fix carbon dioxide from the atmosphere to maintain an optimal growth status. The chlorosis observed in mature leaves might explain this result because plants tend to maintain steady homeostasis. In our study, VvSUC27 was under the control of the $35 \mathrm{~S}$ promoter; therefore, it might have been expressed in all tissues of the transformants. It was found to act as a SUT in the roots, with more than 2-fold of sugar from outside MS sucrose medium in the excess of CK. The sugar concentration decreased in the stems and leaves, indicating that plants can regulate their sugar content within an appropriate range for optimal growth. When grown on medium without sucrose, the sugar content of the plants was considerably lower than that when grown on the sucrose medium; further, no consistent differences were found between the transformants and CK. Furthermore, 
when sucrose in MS media was nearly exhausted after the plants had been grown over 8-9 weeks, the color of the leaves from transformants changed to green (data not shown). However, the transformants planted on the no-sucrose MS medium showed little difference in their sugar content (Figure 8) and in the expression of $l$ hcbs (Figure 7C), compared to those grown on MS medium containing sucrose.

JA, MeJA, ABA, and SA are the key signaling compounds involved in the responses of plants to biotic and abiotic stresses, as well as in development (Wasternack, 2014; Shahzad et al., 2015). Enhanced plant growth characteristics have been attributed to increased IAA and a marked decrease in ABA (Shahzad et al., 2015). Stress-mediating hormones (MeJA, ABA, and SA) are induced in response to mechanical wounding, insect herbivory, and other abiotic and biotic stresses (Santner and Estelle, 2009). However, this beneficial effect is often offset by slowed growth (Shahzad et al., 2015). In our study, the concentration of most of the stress-mediating hormones (MeJA, ABA, and SA) decreased, whereas that of the growth-promoting hormones increased $\left(\mathrm{GA}_{3}\right.$ and IAA) in the transformants grown on the sucrose MS medium (Figure 9). Therefore, we inferred that VvSUC27 promoted the growth of the transformants, leading to an increase in the levels of growthpromoting hormones, which antagonized the effects of the stressmediating hormones. Furthermore, this rapid growth inhibited the synthesis of stress-mediating hormones. The phytohormones of transformants planted on the no-sucrose MS medium were indistinct from those of $\mathrm{CK}$.

Sugars can affect the ribosomal protein paralog composition of ribosomes so that they act as regulatory signals that could affect gene translational activity (Hummel et al., 2012). The changes in sugar levels might affect the activity and expression of the sugar transporter. In citrus (Yao Li et al., 2003), the expression of sucrose transporters was suppressed at high concentrations of sugar. However, in rice embryo, OsSUT1 expression was enhanced by the increased levels of endogenous sugar, as well as light exposure (Matsukura et al., 2000). In the present study, all the transformants had higher expression of VvSUC27 (Figure 10A). In the sucrose medium, the expression of NtSUT1 was significantly suppressed in the roots of transformants, but markedly enhanced in the stems (Figure 10B). However, in the leaves, the expression of NtSUT1 decreased inconspicuously (Figure 10B), probably because the sucrose concentration in the leaves of transformants did not vary as much as that in CK. The transgenic tobacco plants might have attempted to restrict the amount of sucrose taken up via the roots and restricted the expression of NtSUT1. Further, abundant sucrose needs to be transported from the root to the other organs, and the expression of NtSUT1 in the stem was thus enhanced. Thus, NtSUT1 is essential for sucrose export, even when sugar biosynthesis was reduced or soluble sugars accumulated (Burkle et al., 1998). In this study, we compared the expression level of NtSUT1 in medium with and without sucrose and found that NtSUT1 was highly upregulated in the presence of low levels of exogenous sucrose (Figure 10B), which was consistent with the findings for AtSUC9 in Arabidopsis (Jia et al., 2015). When grown in the no-sucrose medium, the expression of NtSUT1 in the transformants was slightly reduced in the roots; however, it was increased in the stems and leaves (Figure 10B). Compared with that in $\mathrm{CK}$, the transformants might have attempted to increase the uptake of sucrose via the roots in the no-sucrose condition; therefore, it released the expression of its NtSUT1. Since, high levels of sucrose were not absorbed, no transport from the root to the other organs was needed. Thus, in the stem, there was no need for enhanced expression of NtSUT1.

\section{VvSUC27 Plays an Important Role under Multiple Abiotic Stresses In vitro}

Source-sink sucrose distribution is known to be beneficial for plant growth and stress tolerance (Roitsch, 1999). Thus far, results on the role of sucrose transporters at the whole-plant level under abiotic stress conditions have revealed a sucrose imbalance in the sink and source, and hypersensitivity in response to abiotic stress treatments when they were induced by defective SUT function, including AtSUC2, AtSUC4, AtSUC9, and PtaSUT4 (Frost et al., 2012; Gong et al., 2015; Jia et al., 2015). This suggests that sucrose transporters might be involved in the redistribution of sucrose, which is useful for plants to resist abiotic stress. To confirm the positive role of VvSUC27 in resistance to abiotic stress, we planted transformants and $\mathrm{CK}$ on medium with and without sucrose under stress conditions. The transformants grown on both sucrose and no-sucrose MS medium showed a stronger phenotype to $\mathrm{CK}$ (Figure 11). ABF acts as an ABAresponsive signaling protein to activate antioxidant defense mechanisms in plants (Ji et al., 2013). ROS levels increase immediately when chelated under abiotic stress, and their scavenging is thought to contribute toward enhancing abiotic stress tolerance in plants (Huang et al., 2010; Yoshida et al., 2015). Therefore, we investigated the transcriptional levels of nine ROS scavengers and ABA-related genes of transformants and CK grown under control or abiotic stress conditions (Figure 12 and Table S2). Most of the genes in the VvSUC27 overexpression lines were significantly up-regulated under stress conditions, especially on sucrose MS medium, and markedly changes could indeed be found in the sucrose content of different parts (Figure 8B) and in the transcriptional levels of nine ROS scavengers and ABA-related genes (Figure 12A), compared with CK in the normal condition. Thus, we inferred that the enhanced stress resistance of VvSUC27 overexpressing lines might be involved in plant abiotic stress tolerance largely dependent on regulating the sucrose content in the source and sink, which might be related to the ABA signaling pathway and to ROS scavenging enzymes.

\section{AUTHOR CONTRIBUTIONS}

YC, JL, and YLZ designed the research. YC, WT, YYZ, JY, and ZX performed the research. YC and YLZ drafted the article.

\section{FUNDING}

This work was supported by the National Natural Science Foundation of China (Grant No. 30900969) and China Agricultural University Scientific Fund (Grant No. 2017sp001). 


\section{ACKNOWLEDGMENTS}

The authors thank Dr. Yunxiao Liu (China Agricultural University) for providing pUC19-CaMV 35S-GFP vector.

\section{REFERENCES}

Afoufa-Bastien, D., Medici, A., Jeauffre, J., Coutos-Thevenot, P., Lemoine, R., Atanassova, R., et al. (2010). The Vitis vinifera sugar transporter gene family: phylogenetic overview and macroarray expression profiling. BMC Plant Biol. 10:245. doi: 10.1186/1471-2229-10-245

Ageorges, A., Issaly, R., Picaud, S., Delrot, S., and Romieu, C. (2000). Identification and functional expression in yeast of a grape berry sucrose carrier. Plant Physiol. Biochem. 38, 177-185. doi: 10.1016/S0981-9428(00)00730-0

Aoki, N., Hirose, T., Takahashi, S., Ono, K., Ishimaru, K., and Ohsugi, R. (1999). Molecular cloning and expression analysis of a gene for a sucrose transporter in maize (Zea mays L.). Plant Cell Physiol. 40, 1072-1078. doi: 10.1093/oxfordjournals.pcp.a029489

Bitterlich, M., Krugel, U., Boldt-Burisch, K., Franken, P., and Kuhn, C. (2014). The sucrose transporter SISUT2 from tomato interacts with brassinosteroid functioning and affects arbuscular mycorrhiza formation. Plant J. 78, 877-889. doi: $10.1111 /$ tpj. 12515

Burkle, L., Hibberd, J. M., Quick, W. P., Kuhn, C., Hirner, B., and Frommer, W. B. (1998). The H+-sucrose cotransporter NtSUT1 is essential for sugar export from tobacco leaves. Plant Physiol. 118, 59-68. doi: 10.1104/pp.118.1.59

Chiou, T. J., and Bush, D. R. (1998). Sucrose is a signal molecule in assimilate partitioning. Proc. Natl. Acad. Sci. U.S.A. 95, 4784-4788. doi: 10.1073/pnas.95.8.4784

Cho, Y. H., and Yoo, S. D. (2011). Signaling role of fructose mediated by FINS1/FBP in Arabidopsis thaliana. PLoS Genet. 7:e1001263. doi: 10.1371/journal.pgen.1001263

Davies, C., Wolf, T., and Robinson, S. P. (1999). Three putative sucrose transporters are differentially expressed in grapevine tissues. Plant Sci. 147, 93-100. doi: 10.1016/S0168-9452(99)00059-X

Deluc, L. G., Grimplet, J., Wheatley, M. D., Tillett, R. L., Quilici, D. R., Osborne, C., et al. (2007). Transcriptomic and metabolite analyses of Cabernet Sauvignon grape berry development. BMC Genomics 8:429. doi: 10.1186/1471-2164-8-429

Deol, K. K., Mukherjee, S., Gao, F., Brule-Babel, A., Stasolla, C., and Ayele, B. T. (2013). Identification and characterization of the three homeologues of a new sucrose transporter in hexaploid wheat (Triticum aestivum L.). BMC Plant Biol. 13:181. doi: 10.1186/1471-2229-13-181

Derrer, C., Wittek, A., Bamberg, E., Carpaneto, A., Dreyer, I., and Geiger, D. (2013). Conformational changes represent the rate-limiting step in the transport cycle of maize sucrose transporter1. Plant Cell 25, 3010-3021. doi: $10.1105 /$ tpc.113.113621

Frost, C. J., Nyamdari, B., Tsai, C. J., and Harding, S. A. (2012). The tonoplastlocalized sucrose transporter in Populus (PtaSUT4) regulates whole-plant water relations, responses to water stress, and photosynthesis. PLoS ONE 7:e44467. doi: 10.1371/journal.pone.0044467

Gabriel-Neumann, E., Neumann, G., Leggewie, G., and George, E. (2011). Constitutive overexpression of the sucrose transporter SoSUT1 in potato plants increases arbuscular mycorrhiza fungal root colonization under high, but not under low, soil phosphorus availability. J. Plant Physiol. 168, 911-919. doi: 10.1016/j.jplph.2010.11.026

Gibson, S. I. (2005). Control of plant development and gene expression by sugar signaling. Curr. Opin. Plant Biol. 8, 93-102. doi: 10.1016/j.pbi.2004.11.003

Gong, X., Liu, M., Zhang, L., Ruan, Y., Ding, R., Ji, Y., et al. (2015). Arabidopsis AtSUC2 and AtSUC4, encoding sucrose transporters, are required for abiotic stress tolerance in an ABA-dependent pathway. Physiol. Plant. 153, 119-136. doi: $10.1111 / \mathrm{ppl} .12225$

Graybosch, R., Hellmann, G. M., Shaw, J. G., Rhoads, R. E., and Hunt, A. G. (1989). Expression of a potyvirus non-structural protein in

\section{SUPPLEMENTARY MATERIAL}

The Supplementary Material for this article can be found online at: http://journal.frontiersin.org/article/10.3389/fpls.2017. 01069/full\#supplementary-material

transgenic tobacco. Biochem. Biophys. Res. Commun. 160, 425-432. doi: 10.1016/0006-291X(89)92450-9

Hossain, M. A., Munemasa, S., Uraji, M., Nakamura, Y., Mori, I. C., and Murata, Y. (2011). Involvement of endogenous abscisic acid in methyl jasmonateinduced stomatal closure in Arabidopsis. Plant Physiol. 156, 430-438. doi: $10.1104 / p p .111 .172254$

Huang, X. S., Liu, J. H., and Chen, X. J. (2010). Overexpression of PtrABF gene, a bZIP transcription factor isolated from Poncirus trifoliata, enhances dehydration and drought tolerance in tobacco via scavenging ROS and modulating expression of stress-responsive genes. BMC Plant Biol. 10:230. doi: 10.1186/1471-2229-10-230

Hummel, M., Cordewener, J. H., de Groot, J. C., Smeekens, S., America, A. H., and Hanson, J. (2012). Dynamic protein composition of Arabidopsis thaliana cytosolic ribosomes in response to sucrose feeding as revealed by label free MSE proteomics. Proteomics 12, 1024-1038. doi: 10.1002/pmic.201100413

Jang, J. C., and Sheen, J. (1994). Sugar sensing in higher plants. Plant Cell 6, 1665-1679. doi: 10.1105/tpc.6.11.1665

Ji, X., Liu, G., Liu, Y., Zheng, L., Nie, X., and Wang, Y. (2013). The bZIP protein from Tamarix hispida, ThbZIP1, is ACGT elements binding factor that enhances abiotic stress signaling in transgenic Arabidopsis. BMC Plant Biol. 13:151. doi: 10.1186/1471-2229-13-151

Jia, W., Zhang, L., Wu, D., Liu, S., Gong, X., Cui, Z., et al. (2015). Sucrose Transporter AtSUC9 mediated by a low sucrose level is involved in Arabidopsis abiotic stress resistance by regulating sucrose distribution and ABA accumulation. Plant Cell Physiol. 56, 1574-1587. doi: 10.1093/pcp/pcv082

Kim, K., Jeong, J., Kim, J., Lee, N., Kim, M. E., Lee, S., et al. (2016). PIF1 regulates plastid development by repressing photosynthetic genes in the endodermis. Mol. Plant 9, 1415-1427. doi: 10.1016/j.molp.2016.08.007

Kuhn, C., Quick, W. P., Schulz, A., Riesmeier, J. W., Sonnewald, U., and Frommer, W. B. (1996). Companion cell-specific inhibition of the potato sucrose transporter SUT1. Plant Cell Environ. 19, 1115-1123. doi: 10.1111/j.1365-3040.1996.tb00426.x

Laby, R. J., Kincaid, M. S., Kim, D., and Gibson, S. I. (2000). The Arabidopsis sugarinsensitive mutants sis 4 and sis 5 are defective in abscisic acid synthesis and response. Plant J. 23, 587-596. doi: 10.1046/j.1365-313x.2000.00833.x

Lastdrager, J., Hanson, J., and Smeekens, S. (2014). Sugar signals and the control of plant growth and development. J. Exp. Bot. 65, 799-807. doi: 10.1093/jxb/ert474

Leggewie, G., Kolbe, A., Lemoine, R., Roessner, U., Lytovchenko, A., Zuther, E., et al. (2003). Overexpression of the sucrose transporter SoSUT1 in potato results in alterations in leaf carbon partitioning and in tuber metabolism but has little impact on tuber morphology. Planta 217, 158-167. doi: 10.1007/s00425-003-0975-x

Lemoine, R. (2000). Sucrose transporters in plants: update on function and structure. Biochim. Biophys. Acta 1465, 246-262. doi: 10.1016/S0005-2736(00)00142-5

Lemoine, R., Kuhn, C., Thiele, N., Delrot, S., and Frommer, W. B. (1996). Antisense inhibition of the sucrose transporter in potato: effects on amount and activity. Plant Cell Environ. 19, 1124-1131. doi: 10.1111/j.1365-3040.1996.tb00427.x

Leterrier, M., Atanassova, R., Laquitaine, L., Gaillard, C., Coutos-Thevenot, P., and Delrot, S. (2003). Expression of a putative grapevine hexose transporter in tobacco alters morphogenesis and assimilate partitioning. J. Exp. Bot. 54, 1193-1204. doi: 10.1093/jxb/erg119

Manning, K., Davies, C., Bowen, H. C., and White, P. J. (2001). Functional characterization of two ripening-related sucrose transporters from grape berries. Ann. Bot. 87, 125-129. doi: 10.1006/anbo.2000.1316

Matsukura, C., Saitoh, T., Hirose, T., Ohsugi, R., Perata, P., and Yamaguchi, J. (2000). Sugar uptake and transport in rice embryo. Expression of companion 
cell-specific sucrose transporter (OsSUT1) induced by sugar and light. Plant Physiol. 124, 85-93. doi: 10.1104/pp.124.1.85

Nowak, W., Gawlowska, M., Jarmolowski, A., and Augustyniak, J. (2001). Effect of nuclear matrix attachment regions on transgene expression in tobacco plants. Acta Biochim. Pol. 48, 637-646.

Oner-Sieben, S., Rappl, C., Sauer, N., Stadler, R., and Lohaus, G. (2015). Characterization, localization, and seasonal changes of the sucrose transporter FeSUT1 in the phloem of Fraxinus excelsior. J. Exp. Bot. 66, 4807-4819. doi: $10.1093 / \mathrm{jxb} / \mathrm{erv} 255$

Pan, X., Welti, R., and Wang, X. (2010). Quantitative analysis of major plant hormones in crude plant extracts by high-performance liquid chromatographymass spectrometry. Nat. Protoc. 5, 986-992. doi: 10.1038/nprot. 2010.37

Pastenes, C., Villalobos, L., Rios, N., Reyes, F., Turgeon, R., and Franck, N. (2014). Carbon partitioning to berries in water stressed grapevines: the role of active transport in leaves and fruits. Environ. Exp. Bot. 107, 154-166. doi: 10.1016/j.envexpbot.2014.06.009

Riesmeier, J. W., Willmitzer, L., and Frommer, W. B. (1992). Isolation and characterization of a sucrose carrier Cdna from spinach by functional expression in yeast. EMBO J. 11, 4705-4713.

Roitsch, T. (1999). Source-sink regulation by sugar and stress. Curr. Opin. Plant Biol. 2, 198-206. doi: 10.1016/S1369-5266(99)80036-3

Rosche, E., Blackmore, D., Tegeder, M., Richardson, T., Schroeder, H., Higgins, T. J. V., et al. (2002). Seed-specific overexpression of a potato sucrose transporter increases sucrose uptake and growth rates of developing pea cotyledons. Plant J. 30, 165-175. doi: 10.1046/j.1365-313X.2002.01282.x

Santner, A., and Estelle, M. (2009). Recent advances and emerging trends in plant hormone signalling. Nature 459, 1071-1078. doi: 10.1038/nature08122

Shahzad, R., Waqas, M., Khan, A. L., Hamayun, M., Kang, S. M., and Lee, I. J. (2015). Foliar application of methyl jasmonate induced physio-hormonal changes in Pisum sativum under diverse temperature regimes. Plant Physiol. Biochem. 96, 406-416. doi: 10.1016/j.plaphy.2015.08.020

Somani, B. L., Khanade, J., and Sinha, R. (1987). A modified anthrone-sulfuric acid method for the determination of fructose in the presence of certain proteins. Anal. Biochem. 167, 327-330. doi: 10.1016/0003-2697(87)90172-2

Sugiura, D., Betsuyaku, E., and Terashima, I. (2015). Manipulation of the hypocotyl sink activity by reciprocal grafting of two Raphanus sativus varieties: its effects on morphological and physiological traits of source leaves and whole-plant growth. Plant Cell Environ. 38, 2629-2640. doi: 10.1111/pce. 12573

Trapp, M. A., De Souza, G. D., Rodrigues, E., Boland, W., and Mithofer, A. (2014). Validated method for phytohormone quantification in plants. Front. Plant Sci. 5:417. doi: 10.3389/fpls.2014.00417

Ueda, T., Yamaguchi, M., Uchimiya, H., and Nakano, A. (2001). Ara6, a plantunique novel type Rab GTPase, functions in the endocytic pathway of Arabidopsis thaliana. EMBO J. 20, 4730-4741. doi: 10.1093/emboj/20.17.4730

Wang, L., Cai, Y., Zhu, L., Guo, H., and Yu, B. (2014). Major role of NADdependent lactate dehydrogenases in the production of l-lactic acid with high optical purity by the thermophile Bacillus coagulans. Appl. Environ. Microbiol. 80, 7134-7141. doi: 10.1128/AEM.01864-14
Wasternack, C. (2014). Action of jasmonates in plant stress responses and development-applied aspects. Biotechnol. Adv. 32, 31-39. doi: 10.1016/j.biotechadv.2013.09.009

Weber, H., Borisjuk, L., Heim, U., Sauer, N., and Wobus, U. (1997). A role for sugar transporters during seed development: molecular characterization of a hexose and a sucrose carrier in fava bean seeds. Plant Cell 9, 895-908. doi: $10.1105 /$ tpc.9.6.895

Weichert, N., Saalbach, I., Weichert, H., Kohl, S., Erban, A., Kopka, J., et al. (2010). Increasing sucrose uptake capacity of wheat grains stimulates storage protein synthesis. Plant Physiol. 152, 698-710. doi: 10.1104/pp.109.150854

Xiang, J., Li, X., Wu, J., Yin, L., Zhang, Y., and Lu, J. (2016). Studying the mechanism of Plasmopara viticola RxLR effectors on suppressing plant immunity. Front. Microbiol. 7:709. doi: 10.3389/fmicb.2016.00709

Xu, F., Xi, Z. M., Zhang, H., Zhang, C. J., and Zhang, Z. W. (2015). Brassinosteroids are involved in controlling sugar unloading in Vitis vinifera "Cabernet Sauvignon" berries during veraison. Plant Physiol. Biochem. 94, 197-208. doi: 10.1016/j.plaphy.2015.06.005

Yao Li, C., Shi, J. X., Weiss, D., and Goldschmidt, E. E. (2003). Sugars regulate sucrose transporter gene expression in citrus. Biochem. Biophys. Res. Commun. 306, 402-407. doi: 10.1016/S0006-291X(03)00978-1

Yoshida, T., Fujita, Y., Maruyama, K., Mogami, J., Todaka, D., Shinozaki, K., et al. (2015). Four Arabidopsis AREB/ABF transcription factors function predominantly in gene expression downstream of SnRK2 kinases in abscisic acid signalling in response to osmotic stress. Plant Cell Environ. 38, 35-49. doi: $10.1111 /$ pce. 12351

Zanon, L., Falchi, R., Hackel, A., Kuhn, C., and Vizzotto, G. (2015). Expression of peach sucrose transporters in heterologous systems points out their different physiological role. Plant Sci. 238, 262-272. doi: 10.1016/j.plantsci.2015.06.014

Zhang, S., Gan, Y., and Xu, B. (2016). Application of plant-growth-promoting fungi Trichoderma longibrachiatum T6 enhances tolerance of wheat to salt stress through improvement of antioxidative defense system and gene expression. Front. Plant Sci. 7:1405. doi: 10.3389/fpls.2016.01405

Zhang, X. Y., Wang, X. L., Wang, X. F., Xia, G. H., Pan, Q. H., Fan, R. C., et al. (2006). A shift of Phloem unloading from symplasmic to apoplasmic pathway is involved in developmental onset of ripening in grape berry. Plant Physiol. 142, 220-232. doi: 10.1104/pp.106.081430

Zhang, Y. L., Meng, Q. Y., Zhu, H. L., Guo, Y., Gao, H. Y., Luo, Y. B., et al. (2008). Functional characterization of a LAHC sucrose transporter isolated from grape berries in yeast. Plant Growth Regul. 54, 71-79. doi: 10.1007/s10725-007-9226-7

Conflict of Interest Statement: The authors declare that the research was conducted in the absence of any commercial or financial relationships that could be construed as a potential conflict of interest.

Copyright (๑) $2017 \mathrm{Cai}, T u, Z u$, Yan, Xu, Lu and Zhang. This is an open-access article distributed under the terms of the Creative Commons Attribution License (CC BY). The use, distribution or reproduction in other forums is permitted, provided the original author(s) or licensor are credited and that the original publication in this journal is cited, in accordance with accepted academic practice. No use, distribution or reproduction is permitted which does not comply with these terms. 